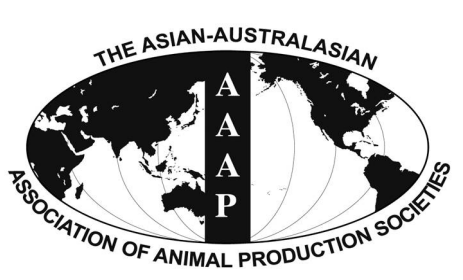

Open Access

Asian Australas. J. Anim. Sci.

Vol. 28, No. 11 : 1657-1661 November 2015

http://dx.doi.org/10.5713/ajas.15.0359

www.ajas.info

pISSN 1011-2367 elSSN 1976-5517

\title{
Heterophil Phagocytic Activity Stimulated by Lactobacillus salivarius L61 and L55 Supplementation in Broilers with Salmonella Infection
}

\author{
Pairat Sornplang*, Vichai Leelavatcharamas', and Chaiyaporn Soikum \\ Department of Veterinary Public Health, Khon Kaen University, Khon Kaen 40002, Thailand
}

\begin{abstract}
Newborn chicks are susceptible to Salmonella enterica serovar Enteritidis (SE). The objective of this study was to evaluate the effect of Lactobacillus probiotic isolated from chicken feces on heterophil phagocytosis in broiler chicks. A total of 150 newborn broiler chicks were divided into 5 groups ( 30 chicks per group) as follows: group 1 (normal control), given feed and water only, group 2 (positive control) given feed, water and SE infection, group 3 (L61 treated) given feed, water, SE infection followed by Lactobacillus salivarius L61 treatment, group 4 (L55 treated) given feed, water, SE infection followed by L. salivarius L55 treatment, and group 5 given feed, water, SE infection followed by L. salivarius L61 + L55 combination treatment. After SE infection, L. salivarius treatment lasted for 7 days. The results showed that L. salivarius L61 and L. salivarius L55 treatment, either alone or combination of both, increased the survival rate after SE infection, and upregulated heterophil phagocytosis and phagocytic index (PI). Conversely, chick groups treated with Lactobacillus showed lower SE recovery rate from cecal tonsils than that of the positive control group. The PI values of the chicken group with SE infection, followed by the combination of L. salivarius L61 and L. salivarius L55 were the highest as compared to either positive control or normal control group. Two Lactobacillus strains supplementation group showed significantly $(\mathrm{p}<0.05)$ higher PI value at $48 \mathrm{~h}$ than $24 \mathrm{~h}$ after treatment. (Key Words: Heterophil, Lactobacillus, Salmonella Enteritidis, Broiler Chicks)
\end{abstract}

\section{INTRODUCTION}

Foodborne illness is usually caused by pathogenic bacteria contamination in food of animal origin such as Salmonella. Salmonellosis in poultry is mainly caused by Salmonella enterica serovar Enteritidis (SE) that can survive in the digestive tract of poultry for several months and contaminate both meat and eggs. Reduction of Salmonella infection in poultry is very important to minimize the loss of chicks and to prevent contamination of meat. Due to an overuse of antibiotic to treat diseases in animals, pathogens have developed a high resistance to antibiotics. Salmonella isolated from chicken has been reported resistant to many antibiotics such as tetracycline, erythromycin, and colistin (Cardoso et al., 2006). To avoid

\footnotetext{
* Corresponding Author: Pairat Sornplang. Tel: +66-43-364493, Fax: +66-43-364493, E-mail: pirson@kku.ac.th

${ }^{1}$ Fermentation research center for value added agricultural products, Khon Kaen University, Khon Kaen 40002, Thailand. Submitted Apr. 24, 2015; Revised Jun. 1, 2015; Accepted Jun. 24, 2015
}

antibiotic residue in meat for consumption, animal rearing has turned to the use of alternatives to antibiotics, such as herbs and probiotics. The largest group of microorganisms used for probiotics is lactic acid-producing bacteria, such as Lactobacillus which is a normal flora found in the gastrointestinal tract of animals including humans (Gilliland et al., 1975). It has been recognized as a safe alternative. To qualify Lactobacillus strains as probiotics, in vivo studies are necessary to clarify their basic properties including survival in the gut environment, antagonistic property to other intestinal pathogens and the ability to adhere the intestinal epithelial cells (Nousiainen and Setala, 1998). However, some studies have reported that probiotic supplementation in chicken feed did not affect their growth (O'Dea et al., 2006). Limitation of probiotics used in animals may be due to uncertainty of their source and whether they specific to a particular animal species. Adhesion to animal epithelial cells of the gastrointestinal tract is supposed to determine host-specificity (Piątek et al., 2012). Immune response of broilers to SE infection has 
been reported in chicks aged 1 to 7 days after hatching; it is sensitive to infection and the broilers still may have a high mortality rate (Barrow et al., 1987). However, many studies reported a probiotic effect that reduces SE infection but there still is a lack information regarding some immune responses such as phagocytic activity in broiler chicks (Bai et al., 2013). Therefore, this study investigated the effect of a Lactobacillus strain isolated from chicken feces that had basic probiotic properties and anti- Salmonella activities in vitro (Sornplang and Leelavatcharamas, 2010), on treating of SE infection and also the effect on phagocytic activity of heterophils in broiler chicks.

\section{MATERIALS AND METHODS}

\section{Bactrial strains}

SE was isolated from a broiler chicken farm in Khon Kaen province, Thailand. Lactobacillus salivarius L61 and L. salivarius L55 were isolated from chickens feces, identified by nucleotide sequence analysis of 16S rRNA genes compared to GenBank with $99 \%$ to $100 \%$ similarity (accession number: CP0003233.1) and these strains can inhibit Salmonella growth in vitro, reported in our previous study (Sornplang and Leelavatcharamas, 2010). Both SE and Lactobacillus isolates were kept as freeze dried specimens at $-70^{\circ} \mathrm{C}$ in a freezer in the Department of Veterinary Public Health, Faculty of Veterinary Medicine, Khon Kaen University, Thailand.

\section{Experimental design}

A total of 150 newborn Arbor Acres chicks of mixed sex were randomly divided into 5 groups (6 pens per group and 5 chicks $/ 1.4 \times 1.5 \mathrm{~m} / \mathrm{pen}$ ). Each group was raised in the open house system under natural conditions. Feed and water were provided ad libitum throughout the experimental period. The feeding trial lasted for 7 days. Nutrition provided by feed was as recommended by the National Research Council (1994) with no antibiotics and Salmonella free. Chicks were challenged with SE by oral gavage of $50 \%$ lethal dose $\left(10^{6}\right.$ colony forming unit, CFU in $\left.0.1 \mathrm{~mL}\right)$ (Pascual et al., 1999). The experimental groups were as follows: Group 1 provided feed and water only, group 2 provided water, feed and SE challenged (positive control group), group 3 provided water, feed, SE challenged and one hour later, $L$. salivarius L61 was supplemented by oral gavage at $10^{9} \mathrm{CFU}$ (Chen et al., 2012), group 4 provided water, feed, $\mathrm{SE}$ challenged and one hour later, $L$. salivarius L55 was supplemented by oral gavage at $10^{9} \mathrm{CFU}$ and group 5 provided water, feed, SE challenged and one hour later Lactobacillus L61 combination with L55 strains was supplemented by oral gavage at $10^{7} \mathrm{CFU}$. Twenty four and 48 hours after Lactobacillus gavage, one chick was randomly selected from each of the 6 pens per treatment $(6$ chicks/treatment). Each chick was slaughtered by cervical dislocation and examined for the presence of SE. The experimental protocol was approved by Khon Kaen University Animal Ethics Committee.

\section{SE detection and isolation}

Cecal tonsil samples were dissolved in the tetrathionate enrichment broth (catalog no. 210420, Becton Dickinson, Sparks, Maryland, USA) and streaked on xylose lactose differential agar plate containing novobiocin $25 \mu \mathrm{g} / \mathrm{mL}$ and nalidixic acid $20 \mu \mathrm{g} / \mathrm{mL}$. These plates were incubated at $37^{\circ} \mathrm{C}$ for 24 hours and then checked for SE (Higgins et al., 2007).

\section{Heterophil isolation}

Blood samples were randomly collected from ulnar vein for one chick from each of the 6 pens per treatment $(6$ chicks/treatment) at 24 and 48 hours after the treatment. Heterophils were isolated by the method of Lowry et al. (2005). In brief, $1 \mathrm{~mL}$ of blood sample was collected in a test tube containing ethylene diamine tetra-acetic acid, and red blood cells were sedimented with 3\% Dextran 500 (Amersham Biosciences Co., Ltd., Uppsala, Sweden). The heterophil rich suspension was mixed with a discontinuous Ficoll-Hypaque (Sigma Chemical Co., St. Louis, MO, USA) and centrifugated at $250 \times \mathrm{g}$ for $30 \mathrm{~min}$. The purity of the heterophil suspension consisted of $90 \%$ heterophils and $10 \%$ macrophages and $>95 \%$ viable as determined by microscopic examination of Wright-stained smears.

\section{Heterophil phagocytosis}

Heterophils were isolated from peripheral blood and adjusted to $5 \times 10^{6}$ cells $/ \mathrm{mL}$, combination of live SE of $5 \times 10^{7}$ cells $/ \mathrm{mL}$ (ratio $1: 10$ ). The protocol was as described by Lowry et al. (2005). Briefly, the mixture was centrifuged and incubated at $39^{\circ} \mathrm{C}$ in $5 \% \mathrm{CO}_{2}$ for $1 \mathrm{~h}$. Samples were submerged in an ice bath for 15 min to stop phagocytosis. Samples were pelleted by centrifugation, supernatants were decanted, and cell/bacteria pellets were resuspended in icecold clear RPMI 1640 (Carlsbad, CA, USA) and washed three times by repeating this process. Gentamicin solution was added to remove residual extracellular SE at the second wash. Sediment cells of the blood were smeared on a slide, stained with Wright's stain and examined microscopically. The results were expressed as percentage of heterophils with bacteria and phagocytic index (PI), where PI = (the percentage of heterophils containing bacteria $) \times($ the average number of bacteria per ingesting heterophil). Phagocytosis and PI values were counted from 200 heterophil cells in different microscope fields.

\section{Bacterial preparation}

Stock freeze-dried culture of Lactobacillus was 
Table 1. Number of surviving chicks after Salmonella Enteritidis (SE) challenge and fed Lactobacillus in posthatch chicks

\begin{tabular}{lccc}
\hline \multirow{2}{*}{ Treatments } & \multicolumn{3}{c}{ Numbers of survival chick (\%) } \\
\cline { 2 - 4 } & $24 \mathrm{~h}$ & $48 \mathrm{~h} *$ & $7 \mathrm{~d}^{* *}$ \\
\hline 1. Feed and water only (basal diet, B) & $29 / 30(96.67)$ & $22 / 23(95.65)$ & $14 / 16(87.50)$ \\
2. B+SE challenged (positive control group) & $18 / 30(60.00)$ & $7 / 12(58.33)$ & $0 / 1(0.00)$ \\
3. B+SE+L. salivarius L61 & $29 / 30(96.67)$ & $23 / 23(100)$ & $16 / 17(94.12)$ \\
4. B+SE + L. salivarius L55 & $29 / 30(96.67)$ & $22 / 23(95.65)$ & $15 / 16(93.75)$ \\
5. B+SE+L. salivarius L61+L. salivarius L55 & $30 / 30(100)$ & $24 / 24(100)$ & $18 / 18(100)$ \\
\hline
\end{tabular}

* Total chicks per group were counted at $24 \mathrm{~h}$ survival and excluded from randomly slaughtered for each group of 6 chicks.

** Total chicks per group were counted at $48 \mathrm{~h}$ survival and excluded from randomly slaughtered for each group of 6 chicks

dissolved into $0.4 \mathrm{~mL}$ de Man, Rogosa, and Sharpe (MRS) broth. It was cultured in MRS agar, and then incubated at $37^{\circ} \mathrm{C}$ overnight or for 18 hours and bacterial growth (adjusted to $10^{9} \mathrm{CFU} / \mathrm{mL}$ ) was measured by the Optical Density $\left(\mathrm{OD}_{600}\right)$. Stock culture of SE was inoculated into tryptic soy agar (Difco, Sparks, Maryland, USA) containing nalidixic acid, incubated at $37^{\circ} \mathrm{C}$ for 18 hours and dissolved in $0.1 \%$ peptone water to measure the OD (adjusted to $10^{6}$ $\mathrm{CFU} / \mathrm{mL}$ ).

\section{Statistical analysis}

Observation values of the mortality, SE recovery, phagocytosis and PI of chicks in each group was analyzed by analysis of variance. The means from these values among groups were further analyzed by Tukey HSD test at the 0.05 level of significance in a completely randomized design.

\section{RESULTS AND DISCUSSION}

This study showed that in the chicks group SE challenged without Lactobacillus treating the mortality was $100 \%$ by 7 -day-old. Chick groups with SE challenged and treated by Lactobacillus exhibited significantly higher survival rate at 1,2 , and 7 days post-treatment compared to the SE challenge group and normal control group (Table 1). Chicks with SE challenged and supplemented with two Lactobacillus strains (L61+L55) showed 100\% survival up to 7 days. Kramer et al. (2001) reported that chicks challenged with SE at the age of 1 day survived for 28 days; the mortality rate was higher than the chicks challenged with SE at the age of 7 days. This may be due to the immune system of 1-day-old chicks that are only partially developed as a similar study of Chen et al. (2012) who reported a cytokine stimulating neutrophils function was lower expression in neonate chicks than the older chicks. Heterophils of broiler chicks have been reported to move into the abdomen in significantly increased quantities after SE challenge (Swaggerty et al., 2005). In this study, survival rate of chicks depended on SE recovery in cecal tonsils. Chicks with SE challenge and treated by Lactobacillus groups showed significantly lower SE recovery in cecal tonsils compared to positive control group and group 1 (Table 2). This study showed one chick infected with SE in the non-infected control group (Table 2). This result can be explained by some broiler chicks being naturally contaminated with Salmonella from their environment, such as bedding materials (Gustafson and Kobland, 1984).

Day-of-hatch chicks inoculated with SE had no SE detected in their cecal tonsils when supplemented with probiotics for $24 \mathrm{~h}$ (Higgins et al., 2010) or for $3 \mathrm{~d}$ (Chen et al., 2012) before the SE inoculation. Our study showed a success of the probiotic treating SE infection in chicks that reduced $\mathrm{SE}$ in cecal tonsil when treated by probiotic within $1 \mathrm{~h}$ after the infection. Supplementation of Lactobacillus L61 and Lactobacillus L61 together with Lactobacillus L55 can eliminate SE infection at 48 and $24 \mathrm{~h}$ post-treatment, respectively. Phagocytosis is an innate immunity, first response to body inflammation or infection. In present study, chick heterophils were activated which challenged with SE. Although, some infected chicks may show a heterophil engulfed SE before treating with the Lactobacillus but all phagocytose heterophils engulfed only one SE per one

Table 2. The Salmonella Enteritidis (SE) recovery from cecal tonsils of experimental chicks (6 chicks/group) at $24 \mathrm{~h}$ and $48 \mathrm{~h}$ after treatment

\begin{tabular}{lcc}
\hline \multirow{2}{*}{ Treatments } & Number of chicks with SE found per chicks examined (\%) \\
\cline { 2 - 3 } & $24 \mathrm{~h}$ & $48 \mathrm{~h}$ \\
\hline 1. Feed and water only (basal diet, B) & $0 / 6(0)$ & $1 / 6(16.67)$ \\
2. B+SE challenged (positive control group) & $6 / 6(100)$ & $6 / 6(100)$ \\
3. B+SE + Lactobacillus salivarius L61 & $1 / 6(16.67)$ & $0 / 6(0)$ \\
4. B+SE + L. salivarius L55 & $2 / 6(33.33)$ & $1 / 6(16.67)$ \\
5. B+SE + L. salivarius L61+L. salivarius L55 & $0 / 6(0)$ & $0 / 6(0)$ \\
\hline
\end{tabular}


Table 3. Effects of Lactobacillus treatment on chicks' heterophil phagocytosis at $24 \mathrm{~h}$ and $48 \mathrm{~h}$ after Salmonella Enteritidis (SE) infection

\begin{tabular}{|c|c|c|c|c|c|c|}
\hline \multirow{2}{*}{ Treatments } & \multicolumn{2}{|c|}{ Percentage of heterophils + SE } & \multicolumn{2}{|c|}{ Average SE: heterophil } & \multicolumn{2}{|c|}{ Phagocytic index } \\
\hline & $24 \mathrm{~h}$ & $48 \mathrm{~h}$ & $24 \mathrm{~h}$ & $48 \mathrm{~h}$ & $24 \mathrm{~h}$ & $48 \mathrm{~h}$ \\
\hline 1. Feed and water only (basal diet, B) & 60 & 62 & 1.3 & 1.27 & 78.00 & 78.74 \\
\hline 2. $\mathrm{B}+\mathrm{SE}$ chalenged (positive control group) & 50 & 51 & 1.12 & 1.12 & 56 & 57.12 \\
\hline 3. $\mathrm{B}+\mathrm{SE}+$ Lactobacillus salivarius $\mathrm{L} 61$ & 74 & 75 & 2.30 & 2.29 & $170.20^{*}$ & $171.75^{*}$ \\
\hline 4. $\mathrm{B}+\mathrm{SE}+$ L.salivarius $\mathrm{L} 55$ & 72 & 73 & 2.19 & 2.19 & $157.68^{*}$ & $159.87 *$ \\
\hline 5. $\mathrm{B}+\mathrm{SE}+$ L.salivarius $\mathrm{L} 61+\mathrm{L} 55$ & 91 & 96 & 4.78 & 5.20 & $434.98 *$ & $499.20 * / * *$ \\
\hline
\end{tabular}

* Indicates significant $(\mathrm{p}<0.05)$ differences were observed between normal control, or positive control and treated in each column.

** Indicates significant $(\mathrm{p}<0.05)$ differences were observed between 24 and $48 \mathrm{~h}$ treated in each row.

heterophil (data not shown). Chick groups with SE challenged and treated by Lactobacillus supplementation can significantly improve $(\mathrm{p}<0.05)$ phagocytosis and PI value at 24 and $48 \mathrm{~h}$ after treatment compared to positive control group and normal control group (Table 3). In this study, supplementation of Lactobacillus probiotic at $1 \mathrm{~h}$ post SE challenged can eradicate SE infection in broiler chicks by $24 \mathrm{~h}$ after treatment. This result was different from the study of Higgins et al. (2010) who reported Lactobacillus treatment after $24 \mathrm{~h}$ of Salmonella infection cannot reduce SE in chicks. The results of Lactobacillus supplementation within $1 \mathrm{~h}$ post SE infection of chicks in our study indicated that this was an advantageous time to eliminate the SE infection. Multi-strains or high dose $\left(\geq 10^{6}\right.$ $\mathrm{CFU} / \mathrm{mL}$ ) of probiotic supplementation showed a positive effect on eradication of intestinal infection as a similar report of Khodadad et al. (2013). In this study, chick heterophils were stimulated efficiently during 24 to $48 \mathrm{~h}$ post-treatment. Chick group with SE challenge and treated by combination of Lactobacillus L61 and Lactobacillus L55 exhibited a significantly higher PI value at $48 \mathrm{~h}$ than that the PI value at $24 \mathrm{~h}$ post-treatment $(\mathrm{p}<0.05)$. In addition, supplementation with two strains of Lactobacillus in this study eradicated SE infection in chicks by $48 \mathrm{~h}$ after treatment. This result is due to the greater innate immune response to SE in probiotic-fed chickens. In conclusion, supplementation of Lactobacillus probiotic (Lactobacillus L61 or L55) can treat Salmonella infection in young chicks when supplemented within $1 \mathrm{~h}$ after infection. This result indicates that Lactobacillus probiotic may be used to prevent Salmonella infection in young chicks when supplemented at an optimal time of posthatch to 2-d-old chicks because their heterophils were more stimulated at this time.

\section{CONFLICT OF INTEREST}

We certify that there is no conflict of interest with any financial organization regarding the material discussed in the manuscript.

\section{ACKNOWLEDGMENTS}

The study was supported by Khon Kaen University, Thailand (grant research 2010 of new researcher development). We thank Dr. Yukifumi Nawa for helpful comments on the manuscript.

\section{REFERENCES}

Bai, S. P., A. M. Wu, X. M. Ding, Y. Lei, J. Bai, K. Y. Zhang, and J. S. Chio. 2013. Effects of probiotic-supplemented diets on growth performance and intestinal immune characteristics of broiler chickens. Poult. Sci. 92:663-670.

Barrow, P. A., M. B. Huggins, M. A. Lovell, and J. M. Simpson. 1987. Observations on the pathogenesis of experimental Salmonella typhimurium infection in chickens. Res. Vet. Sci. 42:194-199.

Cardoso, M. O., A. R. Ribeiro, L. R. dos Santos, F. Pilotto, H. L. S. de Moraes, C. T. Pippi Salle, S. L. da Silveira Rocha, and V. P. do Nascimento. 2006. Antibiotic resistance in Salmonella Enteritidis isolated from broiler carcasses. Braz. J. Microbiol. 37:368-371.

Chen, C. Y., H. Y. Tsen, C. L. Lin, B. Yu, and C. S. Chen. 2012. Oral administration of a combination of select lactic acid bacteria strains to reduce the Salmonella invasion and inflammation of broiler chicks. Poult. Sci. 91:2139-2147.

Gilliland, S. E., M. L. Speck, and C. G. Morgan. 1975. Detection of Lactobacillus acidophilus in feces of humans, pigs, and chickens. Appl. Microbiol. 30:541-545.

Gustafson, R. H. and J. D. Kobland. 1984. Factors influencing salmonella shedding in broiler chickens. J. Hyg. Camb. 92:385-394.

Higgins, J. P., S. E. Higgins, A. D. Wolfenden, S. N. Henderson, A. Torres-Rodriguez, J. L. Vicente, B. M. Hargis, and G. Tellez. 2010. Effect of lactic acid bacteria probiotic culture treatment timing on Salmonella Enteritidis in neonatal broilers. Poult. Sci. 89:243-247.

Higgins, S. E., G. F. Erf, J. P. Higgins, S. N. Henderson, A. D. Wolfenden, G. Gaona-Ramirez, and B. M. Hargis. 2007. Effect of probiotic treatment in broiler chicks on intestinal macrophage numbers and phagocytosis of Salmonella Enteritidis by abdominal exudate cells. Poult. Sci. 86:23152321.

Khodadad, A., F. Farahmand, M. Najafi, and M. Shoaran. 2013. 
Probiotics for the treatment of pediatric Helicobacter pylori infection: A randomized double blind clinical trial. Iran J. Pediatr. 23:79-84.

Kramer, J., A. H. Visscher, J. A. Wagenaar, A. G. Boonstra-Blom, and S. H. M. Jeurissen. 2001. Characterization of the innate and adaptive immunity to Salmonella enteritidis PT1 infection in four broiler lines. Vet. Immunol. Immunopathol. 79:219-223.

Lowry, V. K., M. B. Farnell, P. J. Ferro, C. L. Swaggerty, A. Bahl, and M. H. Kogut. 2005. Purified $\beta$-glucan as an abiotic feed additive up-regulates the innate immune response in immature chickens against Salmonella enterica serovar Enteritidis. Int. J. Food Microbiol. 98:309-318.

National Research Council. 1994. Nutrient Requirements of Poultry. 9th edn. National Academy Press, Washington, DC, USA.

Nousiainen, J. and J. Setala. 1998. Lactic acid bacteria as animal probiotics. In: Lactic Acid Bacteria (Eds. S. Salminen and A. von Wright). 2nd edn. Mercel Dekker, New York, USA. pp. 437-473.
O'Dea, E. E., G. M. Fasenko, G. E. Allison, D. R. Korver, G. W. Tannock, and L. L. Guan. 2006. Investigating the effects of commercial probiotics on broiler chick quality and production efficiency. Poult. Sci. 85:1855-1863.

Pascual, M., M. Hugas, J. I. Badiola, J. M. Monfort, and M. Garriga. 1999. Lactobacillus salivarius CTC2197 prevents Salmonella enteritidis colonization in chickens. Appl. Environ. Microbiol. 65:4981-4986.

Piątek, J., M. Gibas-Dorna, A. Olejnik, H. Krauss, K. Wierzbicki, W. Żukiewicz-Sobczak, and M. Głowacki. 2012. The viability and intestinal epithelial cell adhesion of probiotic strain combination: in vitro study. Ann. Agric. Environ. Med. 19:99102 .

Sornplang, P. and V. Leelavatcharamas. 2010. Antimicrobial susceptibility of probiotic lactobacilli isolated from chicken feces. KKU Res. J. 15:689-697.

Swaggerty, C. L., P. J. Ferro, I. Y. Pevzner, and M. H. Kogut. 2005. Heterophils are associated with resistance to systemic Salmonella enteritidis infections in genetically distinct chicken lines. FEMS Immunol. Med. Microbiol. 43:149-154. 